\title{
A Study of Surface Modification of Poly(lactic-co-glycolic) Acid Using Argon Ion Irradiation
}

\author{
Ananta Raj Adhikari1 ${ }^{*}$, Buddhi Prasanga Tilakaratne1, Dharshana Wijesundera1, \\ Wei-Kan Chu1,2 \\ ${ }^{1}$ Texas Center for Superconductivity, University of Houston, Houston, USA \\ ${ }^{2}$ Department of Physics, University of Houston, Houston, USA \\ Email: aa8381@gmail.com
}

Received 24 July 2014; revised 26 August 2014; accepted 16 September 2014

Copyright (C) 2014 by authors and Scientific Research Publishing Inc.

This work is licensed under the Creative Commons Attribution International License (CC BY).

http://creativecommons.org/licenses/by/4.0/

c) (i) Open Access

\section{Abstract}

The effect of Argon ion irradiation to the surface properties of poly(lactic-co-glycolic) acid (PLGA) was studied. A beam of $170 \mathrm{keV}$ Argon ions was implanted at different fluencies $\left(1 \times 10^{12}, 1 \times 10^{13}\right.$, $1 \times 10^{14}$, and $1 \times 10^{15}$ ions $/ \mathrm{cm}^{2}$ ). X-ray photoelectron spectroscopy (XPS) was used to analyze the evolution of the bonding microstructure of PLGA due to irradiation. Surface morphology was monitored using atomic force microscopy (AFM). AFM analysis shows that film roughness increased to maximum at the dose of $1 \times 10^{14}$ ions $/ \mathrm{cm}^{2}$ where the formations of hillocks were also detected. Hydrophilicity of PLGA is important for their applications in biomedicine such as bioscaffolds. Hydrophilicity was monitored using water contact angle measurements for both unmodified and ion-modified PLGA. It was observed that hydrophilicity of PLGA changes with the ion irradiation. This demonstrates that ion irradiation can be an alternative approach to control hydrophilicity of PLGA. PLGA scaffolds modified with ion irradiation could therefore be more suitable for the biomedical applications.

\section{Keywords}

Poly(lactic-co-glycolic) Acid Scaffold, Surface Modification, Ion Irradiation

\section{Introduction}

The advances of science and technology have significantly increased the use of flexible, light weight, and mul-

"Corresponding author.

How to cite this paper: Adhikari, A.R., Tilakaratne, B.P., Wijesundera, D. and Chu, W.-K. (2014) A Study of Surface Modification of Poly(lactic-co-glycolic) Acid Using Argon Ion Irradiation. Journal of Surface Engineered Materials and Advanced Technology, 4, 326-331. http://dx.doi.org/10.4236/isemat.2014.46036 
tifunctional materials. Among the many, the use of biopolymers is gaining more and more advantageous due to their natural abundancy, bioresorbability, and biodegradability. These properties are vital to maintain clean and healthy environment by replacing the petro-based polymer that leaves the threatening alarm to the future world. On the other hand, biopolymers with specific thermal, mechanical, chemical, and morphological properties are anticipated to use in various biomedical applications such as tissue engineering. In soft tissue engineering such as a scaffold, the surface properties of the scaffolds should be designed in order to facilitate the cell growth, proliferation, and attachment and finally provide the healthy living tissues. Besides providing a suitable platform for the cell, the degradation behavior of scaffolds is critical to support the growth cells, transfer cells to the tissues, and finally clear the matrix. These biological processes will be determined by the surface properties of scaffolds such as surface roughness, porosity, and surface chemistry.

Poly(lactic-co-glycolic) acid (PLGA) is one of the food and drug association (FDA) approved biopolymers that are biodgradable and bioresorbable [1] [2]. PLGA is insoluble in water. Due to the presence of ester bond it is susceptible to hydrolytically attack and form natural metabolites (lactic and glycolic acids) that are eliminated through the citric acid cycle [3] [4]. The degradation behavior and mechanical property of PLGA are important for their application in biomedicine. This can be controlled by varying the ratio of lactide and glycolide in the polymer chain, the molecular weight, crystallinity, and the glass transition temperature of PLGA [5] [6]. PLGA in its natural forms is not ideal for applications such as scaffold for cell growth [7]. This is due to its low surface energy and high hydrophobicity compared to the natural extracellular matrix (ECM) resulting in its poor affinity for the cells growth and their attachment.

Tailoring surface energy of PLGA is thus an important step to bring this polymer into biomedicine. Different promising approaches have been adopted to modify surface microstructure of polymer such as mixing with nanofillers [8], biochemical coating [9] or physio-chemical treatment of the surfaces [10]-[13]. Adhikari et al. [8] have utilized the graphene platelets as a stage for polymer crystallization which was shown effective to grow human embryonic kidney cells compared to unmodified polymer. Similarly, Islam et al. [10] demonstrated the use of gamma ray irradiation on hybrid polymer network of chitosan (CS) with poly(vinyl alcohol) (PVA) to modify the hydrophilic nature due to radiation-induced polymer scission and reported the non-toxic nature of modified scaffolds suitable for tissue engineering and other biomedical applications.

The use of irradiation in polymer surface modification is increasing rapidly [10]-[15] by taking advantage of the energy lost by the high energy particles during interaction with the polymer chain. Ion beam interacts with materials by two processes: inelastic scattering responsible for the electronic ionization and excitation known as electronic energy loss, and elastic scattering where there is a direct momentum transfer to target materials known as nuclear energy loss resulting in vacancy formation and interstitials. The degree of damage depends on ion mass, energy, and the fluencies. These processes are observed dominant in polymer. Figure 1 shows the energy loss as a function of depth of PLGA based on SRIM2013 calculation. This clearly demonstrates that at the surface the electronic interaction slightly dominates over the nuclear interaction. The nature of the interaction changes as the energy of the projectile changes. As the energy decreases the nuclear interaction dominates over electronic interaction. Further, ion fluency can be varied to adjust the defects density. This shows the feasibility of using ion irradiation for surface as well as bulk modification by tailoring energy and the size of the ions.

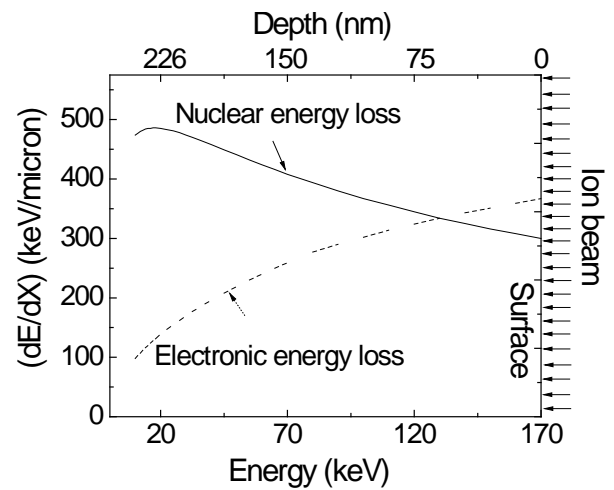

Figure 1. Illustration of $170 \mathrm{keV}$ Argon energy loss within PLGA based on SRIM 2013 calculation. 
The objective of this study is to investigate the role of low energy (170 keV) Argon ion irradiation in the PLGA surface properties. Samples were analyzed for their surface microstructure by using X-ray photoelectron spectroscopy and atomic force spectroscopy before and after irradiation. Similarly, the contact angle measurements were performed to account the nature of hydrophilicity.

\section{Experimental Section}

\section{Materials and Methods}

PLGA was received from Purac Biomaterials. The obtained PLGA consist of poly(lactic acid) (PLA) and poly(glycolic acid) (PGA) in 50:50 molar ratio with inherent viscosity of $1.05 \mathrm{dl} / \mathrm{g}$ (molecular weight about $170,000 \mathrm{gm} / \mathrm{mol}$ ). PLGA was initially stirred in anhydrous Chloroform at room temperature for 24 hours. Thin films of PLGA were then prepared on Silicon substrate by spin cast method. All samples were then kept in oven at $40^{\circ} \mathrm{C}$ for 48 hours to evaporate solvents. Irradiation was performed using ion beam facility ( $200 \mathrm{keV}$ ion implanter) housed in University of Houston. Argon ions of $170 \mathrm{keV}$ were implanted from fluencies $10^{12}$ to $10^{15}$ ions $/ \mathrm{cm}^{2}$ in a chamber pressure maintained at about $2 \times 10^{-5} \mathrm{~Pa}$.

The changes of surface topography were monitored before and after Argon irradiation using atomic force microscopy from Park Scientific Instruments. The contact mode measurements were made in air with a Silicon tip of radius $10 \mathrm{~nm}$. The scan rate and scan lines per images are $0.5 \mathrm{~Hz}$ and 256 respectively. X-ray photoelectron spectroscopy (XPS) measurements were obtained by Physical Electronics Model 5700 using monochromatic Al $\mathrm{K}_{\alpha} \mathrm{X}$-ray source $1486.6 \mathrm{eV}$ operated at $350 \mathrm{~W}$. The analyzed area was set to $1 \mathrm{~mm}$ diameter and take-off angle was set in the range of 30 - 70 degrees. The photoelectrons were allowed to pass through a hemispherical analyzer with a low pass energy filter of $11.75 \mathrm{eV}$, which resulted in energy resolution of $0.51 \mathrm{eV}$. Data was processed using the Multipak software package (Physical Electronics, Inc.). Contact angle measurements were conducted with sessile water drop method using data physics OCA 15EC contact angle goniometer. The drop size that was placed on the surface was approximately $1 \mu \mathrm{L}$ of distilled water. The contact angle dynamics was studied by measuring left and right contact angle of the water drop over a time span of 3 minutes.

\section{Results and Discussion}

Atomic force microscopy (AFM) images and the corresponding rms roughness of as deposited and Argon ion irradiated PLGA is shown in Figure 2. The effects of ion irradiation can be directly visualized from the surface analysis of the matrix such as change of surface roughness and formation of craters/hillocks. Surface topography
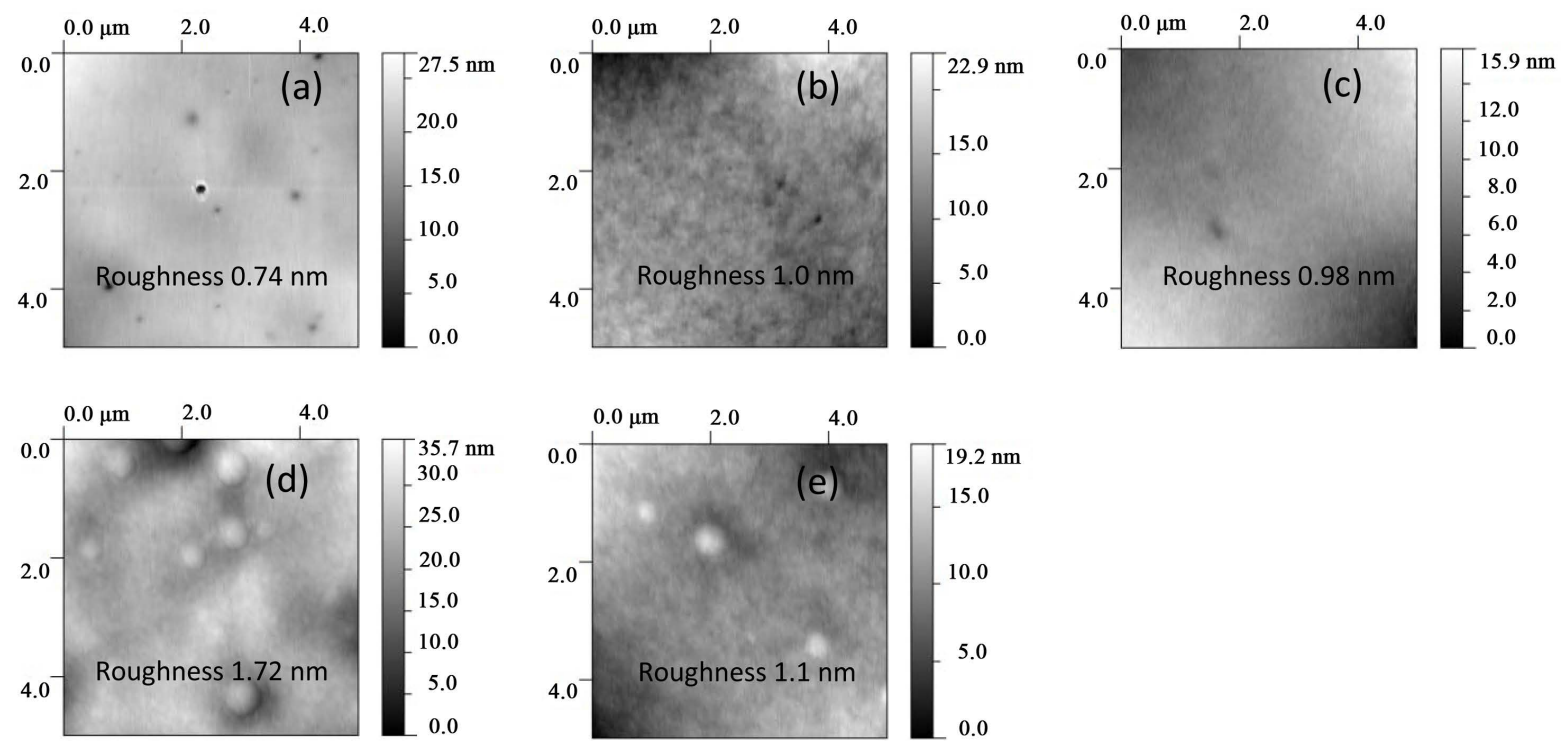

Figure 2. Tapping-mode atomic force microscopy 2-D images of (a) as deposited PLGA and Argon ion irradiated PLGA with doses (b) $1 \times 10^{12}$ ions $/ \mathrm{cm}^{2}$, (c) $1 \times 10^{13} \mathrm{ions} / \mathrm{cm}^{2}$, (d) $1 \times 10^{14} \mathrm{ions} / \mathrm{cm}^{2}$, and (e) $1 \times 10^{15} \mathrm{ions} / \mathrm{cm}^{2}$. 
of unirradiated PLGA (Figure 2(a)) consist of a number of holes, possibly are the process related defects. Figure 2(b) clearly distinguishes the effect of irradiation where the surface roughness increases with lesser numbers of holes compared to holes in the as deposited PLGA. An additional feature (bubbles/hillocks) on the PLGA surface appeared after the Argon fluency of $1 \times 10^{14}$ ions $/ \mathrm{cm}^{2}$ where the surface roughness increases to a maximum value of $1.72 \mathrm{~nm}$ among the samples studied here compared to the roughness $0.74 \mathrm{~nm}$ of as deposited PLGA. The size of hillock was found varied from 0.2 to $0.5 \mu \mathrm{m}$ from the section analysis. At higher Argon dose of $1 \times 10^{15}$ ions $/ \mathrm{cm}^{2}$ the numbers of bubbles/hillocks as well as the surface roughness decreased.

It is well known that ion irradiation deposits energy to the target along the ion track through different processes that lead to the different types of damages to the target. The possible mechanisms are the energy loss through electronic and nuclear interaction of projectile with the target materials. In polymer the increase of roughness is due to the polymer chain scissioning followed by polymer cross linking that also accounts the degradation of polymer. The decrease of roughness for $1 \times 10^{15} \mathrm{Ar}^{+}$ions $/ \mathrm{cm}^{2}$ irradiated PLGA in our case can be taken as the dominating process of amorphization of the polymer due to irradiation. Formation of hillocks and craters in polymer is a well observed phenomenon in ion bombarded polymer. This is due to gas released such as $\mathrm{O}_{2}, \mathrm{CH}_{4}, \mathrm{CO}_{2}$ and $\mathrm{H}_{2}$ from the chain scission in polymer [14]. Based on model by D. He and M. N. Bassim [15] the observed hillocks is due to the diffusion of gases and trapped by defects in the polymer formed during irradiation along the ion track, thus forming bubbles on the surface. The decrease of hillocks number at the dose of $1 \times 10^{15}$ ions $/ \mathrm{cm}^{2}$ could be expected as the coalescence of hillocks and finally breaking of blisters with gas release.

The evolution of surface microstructure of PLGA with the Argon ion was investigated using X-ray photoelectron spectroscopy (XPS). Figure 3(a) shows the C1s spectra of as deposited and Argon implanted PLGA. Plot shows three carbon peaks at $285 \mathrm{eV}$ (Peak 1), $287 \mathrm{eV}$ (Peak 2), and $289 \mathrm{eV}$ (Peak 3). Similar observation was made by Shakesheff et al. [16]. Considering the chemical structure of the copolymer PLGA, Peak 1, Peak 2, and Peak 3 are assigned to methyl group $(\mathrm{C}-\mathrm{H})$, to the neighboring $(\mathrm{C}-\mathrm{O})$ carbon in the backbone, and the carboxylic group $(\mathrm{C}=\mathrm{O})$ carbon of the polymer respectively. C1s spectra of unmodified PLGA (Figure 3(a)) shows Peak 1 with smaller peak area signifies that PLGA consist of glycolide monomer possessing only methylene and ester groups compared to the lactide monomer.

Ion irradiation deposit energy to the polymer through electronic and nuclear energy loss. Electronic energy loss results electronic excitation and ionization. On the other hand, nuclear energy loss is susceptible for permanent bond breakage through elastic scattering that results in polymer chain scission. We observed that $\mathrm{C}-\mathrm{O}$ and $\mathrm{C}=\mathrm{O}$ bonds in polymer is decreasing with irradiation as observed in Figure 3(b) for ion doses higher than $1 \times$
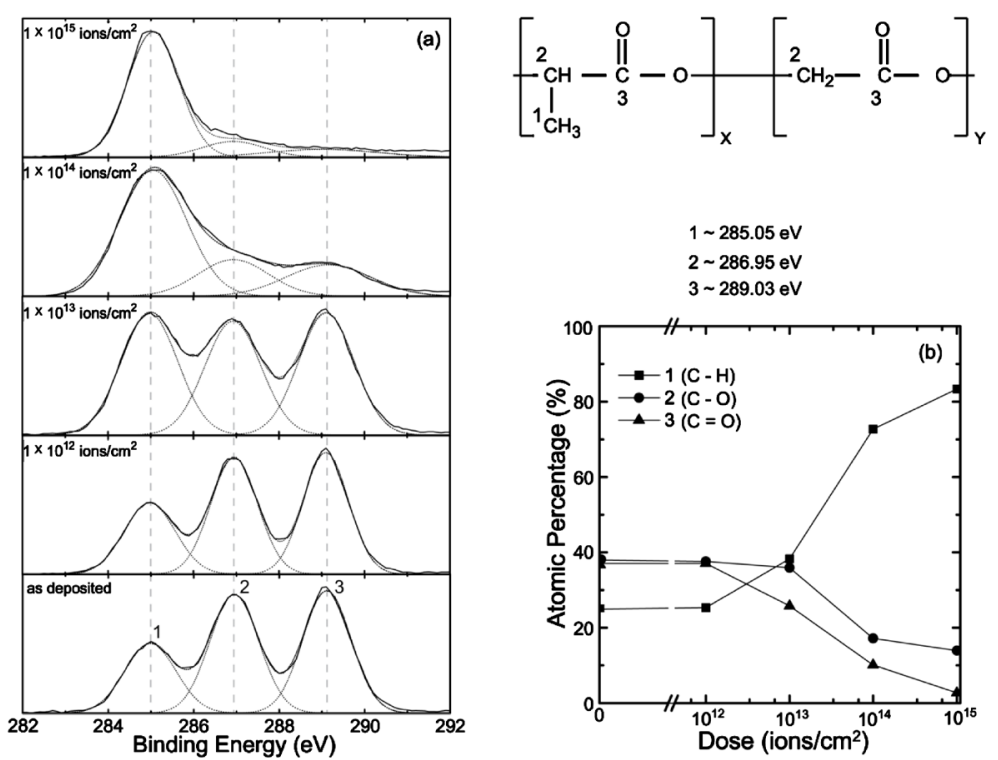

Figure 3. (a) C1s regions of XPS data for PLGA polymer for different Argon ion irradiation; (b) Variation of atomic percentage of (1) C-H, (2) C-O, (3) $\mathrm{C}=\mathrm{O}$ bonding present in the PLGA polymer. 
$10^{12} \mathrm{ions} / \mathrm{cm}^{2}$. This shows that Argon irradiation decreases the peak 2 and peak 3 areas with the increase of ion fluencies. This clearly demonstrates the PLGA chain scission at doses higher than $1 \times 10^{12} \mathrm{ions} / \mathrm{cm}^{2}$.

XPS analysis demonstrated the change of $\mathrm{C}-\mathrm{H}, \mathrm{C}-\mathrm{O}$, and $\mathrm{C}=\mathrm{O}$ bonding in PLGA from the Argon ion interaction. PLGA with different combination of lactide and glycolide exhibits wide range of surface sensitive behavior. In addition, to study the effects of these changes in surface composition of PLGA due to irradiation contact angle measurements were performed to account the hydrophilicity of the unmodified and irradiated PLGA. Figure 4 shows time dependence change in water contact angle of PLGA before and after Argon irradiations. Different Argon treatments were made to tailor the surface microstructures. A significant change of contact angle observed even for the dose of $1 \times 10^{12} \mathrm{Ar}$ ions $/ \mathrm{cm}^{2}$ where there is clear decrease of contact angle. Contact angle further decreased and maintained minimum at the dose of $1 \times 10^{14} \mathrm{Ar}$ ions $/ \mathrm{cm}^{2}$. This shows the increase of surface energy of PLGA at doses smaller than $1 \times 10^{14} \mathrm{Ar}$ ions $/ \mathrm{cm}^{2}$ and decreased thereafter at higher Argon dose (Figure 4 insert). As shown in Figure 1 both the nuclear and electronic interaction compete each other and subsequently generates different types of defects such as vacancy, interstitials, grain boundaries, and atoms with less number of electrons. These are high energy states which are reactive to the environment. Water possesses permanent dipole moment which are susceptible to react with the reactive site containing defects mentioned thereby decreasing the water contact angle. In addition we can predict from these analysis that presence of easter group is an important factor for hydrophilicity. This demonstrates that ion irradiation can be used for tailor the degree of hydrophilicity of the PLGA. The next study will be their degradation studies of these modified PLGA to make use of these defects from irradiation.

\section{Conclusion}

In summary, PLGA was processed using ion irradiations to tailor the surface behavior such as surface roughness and hydrophilicity. Atomic force microscopy images of PLGA allowed us to monitor the surface modification from Argon irradiations. At the Argon dose of $1 \times 10^{14} \mathrm{ions} / \mathrm{cm}^{2}$ the surface roughness (1.7 nm) of PLGA found maximum where the hillocks started to form. X-ray photoelectron spectroscopy observation showed that PLGA surface microstructure can be tailored using Argon irradiation of PLGA. Finally, the water contact angle measurements revealed that Argon irradiation at the dose of $1 \times 10^{14}$ ions $/ \mathrm{cm}^{2}$ decreases the contact angle to about $65^{\circ}$ when compared to unmodified PLGA, demonstrating the increase of hydrophilicity of Argon irradiated PLGA. Such materials with moderate contact angles value $\left(40^{\circ}-70^{\circ}\right)$ are more suitable for their application in biomedicine such as scaffolds that comparatively absorb more cell adhesive protein [17].

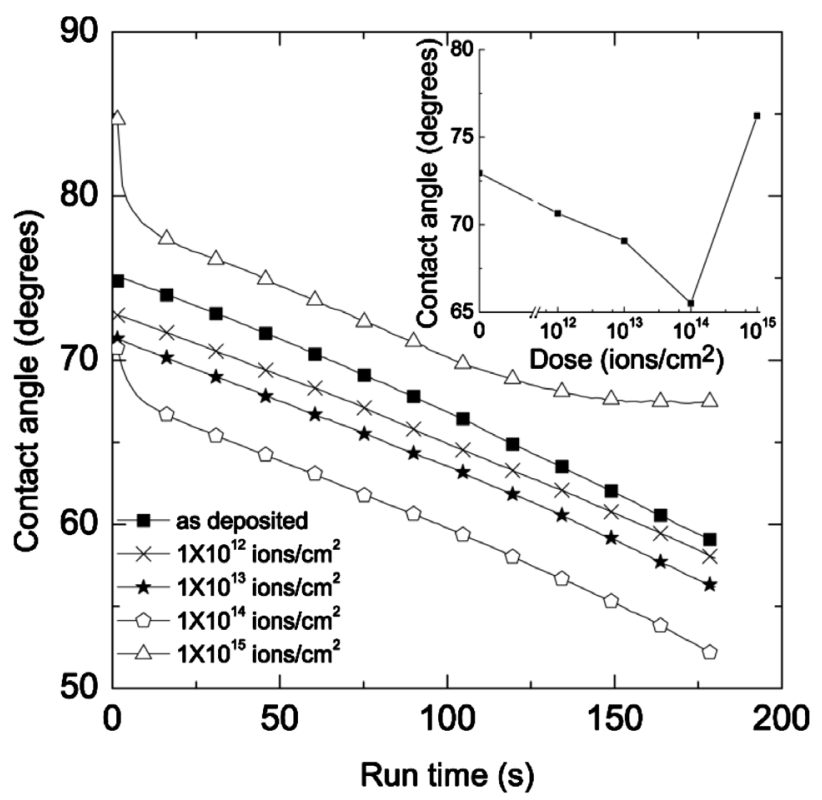

Figure 4. Dynamic contact angle for different Argon ion irradiation. Inset figure was plotted for the change of contact angle as a function of ion doses where the contact angles were taken at $30 \mathrm{sec}$ run time. 


\section{Acknowledgements}

This study was supported by the State of Texas through Texas Center for Superconductivity at University of Houston.

\section{References}

[1] Lee, C.T. and Lee, Y.D. (2006) Preparation of Porous Biodegradable Poly(lactide-co-glycolide)/Hyaluronic Acid Blend Scaffolds: Characterization, in Vitro Cells Culture and Degradation Behaviors. Journal of Materials Science: Materials in Medicine, 17, 1411-1420. http://dx.doi.org/10.1007/s10856-006-0617-5

[2] Jayasuriya, A.C. and Ebraheim, N.A. (2009) Evaluation of Bone Matrix and Demineralized Bone Matrix Incorporated PLGA Matrices for Bone Repair. Journal of Materials Science: Materials in Medicine, 20, 1637-1644. http://dx.doi.org/10.1007/s10856-009-3738-9

[3] Beiser, I.H. and Kanat, I.O. (1990) Biodegradable Internal Fixation. A Literature Review. Journal of the American Podiatric Medical Association, 80, 72-75. http://dx.doi.org/10.7547/87507315-80-2-72

[4] Griffith, L.G. (2000) Polymeric Biomaterials. Acta Materialia, 48, 263-277. http://dx.doi.org/10.1016/S1359-6454(99)00299-2

[5] Wu, L. and Ding, J. (2004) In Vitro Degradation of Three-Dimensional Porous Poly(d,l-lactide-co-glycolide) Scaffolds for Tissue Engineering. Biomaterials, 25, 5821-5830. http://dx.doi.org/10.1016/j.biomaterials.2004.01.038

[6] Wu, X.S. (1995) Encyclopedic Hand Book of Biomaterials, Bioengineering. Marcel Dekker, New York, 1015-1054.

[7] Yang J., Shi, G., Bei, J., Wang, S., Cao, Y., Shang, Q., Yang, G. and Wang, W. (2002) Fabrication and Surface Modification of Macroporous Poly(L-lactic Acid) and Poly(L-lactic-co-glycolic Acid) (70/30) Cell Scaffolds for Human Skin Fibroblast Cell Culture. Journal of Biomedical Materials, 62, 438-446. http://dx.doi.org/10.1002/jbm.10318

[8] Adhikari, A.R., Rusakova, I., Haleh, A., Luisi, J., Panova, N.I., Laezza, F. and Chu, W.K. (2014) Thermal Property and Assessment of Biocompatibility of Poly(lactic-co-glycolic) Acid/Graphene Nanocomposites. Journal of Applied Physics, 115, 054701-054706. http://dx.doi.org/10.1063/1.4864263

[9] Sanchez, A., Alvarez, A., Pagan, R., Roncero, C., Vilarb, S., Benito, M. and Fabregat, I.J. (2000) Fibronectin Regulates Morphology, Cell Organization and Gene Expression of Rat Fetal Hepatocytes in Primary Culture. Hepatology, 32, 242-250. http://dx.doi.org/10.1016/S0168-8278(00)80069-0

[10] Islam, A., Yasin, T. and Rehman, I.U. (2014) Synthesis of Hybrid Polymer Networks of Irradiated Chitosan/Poly(vinyl Alcohol) for Biomedical Applications. Radiation Physics and Chemistry, 96, 115-119. http://dx.doi.org/10.1016/j.radphyschem.2013.09.009

[11] Ginn, B.T. and Steinbock, O. (2003) Polymer Surface Modification Using Microwave-Oven-Generated Plasma. Langmuir, 19, 8117-8118. http://dx.doi.org/10.1021/la034138h

[12] Loo, J.S.C., Ooi, C.P. and Boey, F.Y.C. (2005) Degradation of Poly(lactide-co-glycolide) (PLGA) and Poly(L-lactide) (PLLA) by Electron Beam Radiation. Biomaterials, 26, 1359-1367. http://dx.doi.org/10.1016/j.biomaterials.2004.05.001

[13] Yoshihisa, K., Yoshimura, A., Shibamori, Y., Fuchigami, K. and Kubota, N. (2012) Polymer Surface Modification by Using Microwave Plasma Irradiation. Journal of Solid Mechanics and Material Engineering, 6, 654-659. http://dx.doi.org/10.1299/jmmp.6.654

[14] Khalfaoui, N., Rotaru, C.C., Bouffard, S., Toulemonde, M., Stoquert, J.P., Haas, F., Trautmann, C., Jensen, J. and Dunlop, A. (2005) Characterization of Swift Heavy Ion Tracks in $\mathrm{CaF}_{2}$ by Scanning Force and Transmission Electron Microscopy. Nuclear Instruments and Methods in Physics Research B, 240, 819-828. http://dx.doi.org/10.1016/j.nimb.2005.06.220

[15] He, D. and Bassim, M.N. (1998) Atomic Force Microscope Study of Crater Formation in Ion Bombarded Polymer. Journal of Material Science, 33, 3525-3528. http://dx.doi.org/10.1023/A:1004634724569

[16] Shakesheff, K.M., Evora, C., Soriano, I. and Langer, R. (1997) The Adsorption of Poly(vinyl Alcohol) to Biodegradable Microparticles Studied by X-Ray Photoelectron Spectroscopy (XPS). Journal of Colloid and Interface Science, 185, 538-547. http://dx.doi.org/10.1006/jcis.1996.4637

[17] Arima, Y. and Iwata, H. (2007) Effect of Wettability and Surface Functional Groups on Protein Adsorption and Cell Adhesion Using Well-Defined Mixed Self-Assembled Monolayers. Biomaterials, 28, 3074-3082. http://dx.doi.org/10.1016/j.biomaterials.2007.03.013 
Scientific Research Publishing (SCIRP) is one of the largest Open Access journal publishers. It is currently publishing more than 200 open access, online, peer-reviewed journals covering a wide range of academic disciplines. SCIRP serves the worldwide academic communities and contributes to the progress and application of science with its publication.

Other selected journals from SCIRP are listed as below. Submit your manuscript to us via either submit@scirp.org or Online Submission Portal.
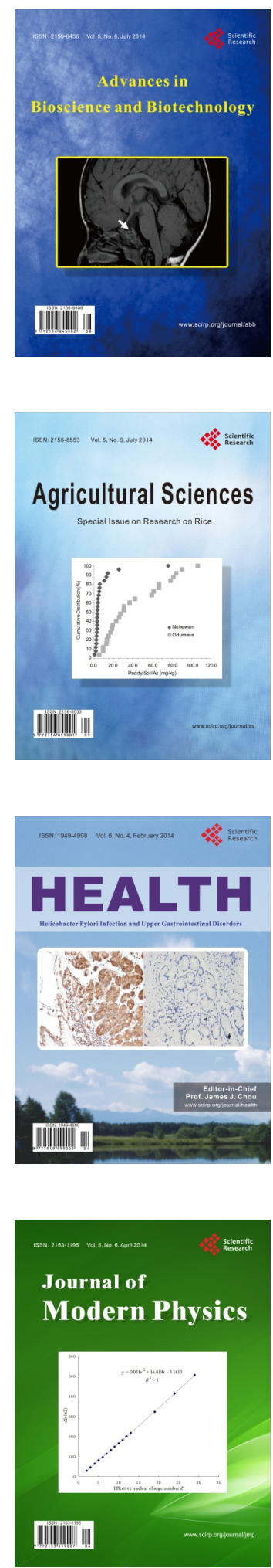
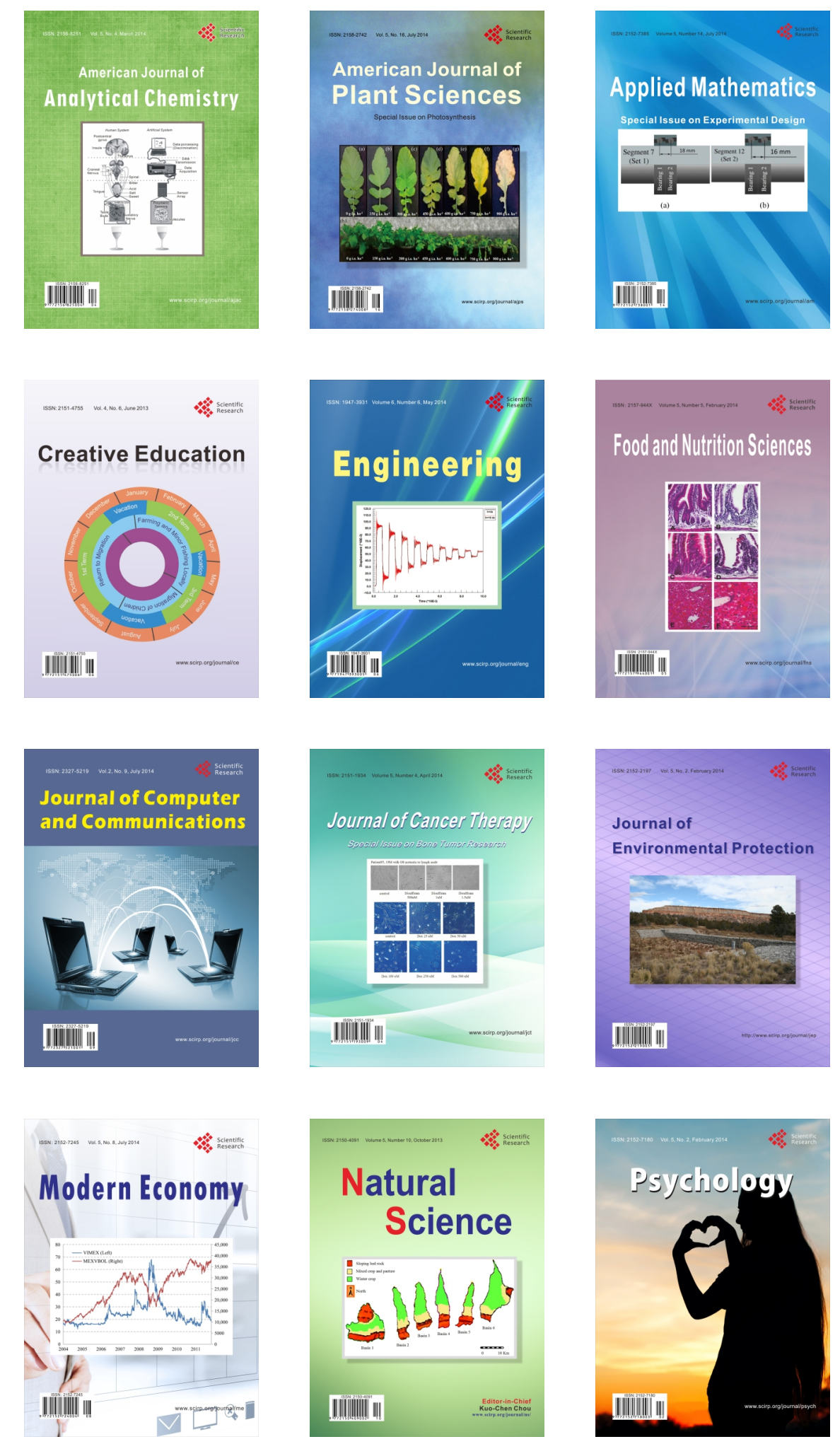